\title{
Korobchanskiy V.O. \\ MEDICINE OF BORDERLINE CONDITIONS IN RETRIEVAL OF NEW HEALTH CARE PARADIGM
}

Kharkiviv National Medical University, Ukraine

In the methodology of science, the term refers to the paradigm accepted by all scientific advances that allow the scientific community for some time to have a model of statement and solution of problems.

Medicine is no exception.

The essence of the health care paradigm is taken in the whole set of knowledge and methodological approaches to the solution of health problems which are approved by society and the professional medical community. At the same time the ways to achieve this goal have changed over the centuries, spanning the entire spectrum of approaches - from empiric to systematic analysis.

Public health strategy was repeatedly revised in the developed countries in the $20^{\text {th }}$ century.

The struggle against epidemic infections lay in its base up to the 1960s, and later to ensure protection against chronic diseases of infectious nature.

Today the focus is transferred not only to the patient or a person at risk group, but also to the reproduction of a healthy population, the care of the birth of a healthy baby.

Developed countries have recently started to consider the interdisciplinary nature of public health protection at the national level. Nowadays the functional health care paradigm formed within these strategies, and the factors and conditions that led to its crisis formed at the same time. 
Summarizing the effects of numerous factors contributing to the change of the current health paradigm and hence the need for its reform we should allocate such directions:

I. Changes in medical, biological and demographic factors, habitat and human mentality.

II. Enhancing the role of socio-economic factors and well-being in forming of public health.

III. The growth of health care costs, the excess of the health funding needs over the possibilities of the state.

IV. Changing the role of health care in the system of public health.

V. Formation of legal and evidence-based medicine, standardization of medical practice.

Formation of a new health care paradigm occurs not only in Ukraine, or in post-socialist countries. This is typical for all countries in the world.

Formation of the new paradigm is always in the framework of the old paradigm. This is due to the emergence of new alternative theories and ideas, when previous consensus on the old paradigm is broken and new factors of external and internal environment necessitate a new paradigm.

We refer methodology of medicine of borderline conditions for such a new alternative theory.

This idea was formulated at our university in 2013 (Medicine of borderline conditions: theory and practice of prenosological diagnosis / V.M. Lesoviy, V.A. Kapustnik, V.O. Korobchansky // Scientific journal of Ministry of Health of Ukraine, 2013. - № 2. (3), p.49 -60). It was founded on the concept of prenozological, which was formulated in the early 1980s by the representatives of the Leningrad scientific medical school.

The idea got creative development in the works of such Ukrainian specialists as A.M. Serdyuk, I.M. Trachtenberg, V.G. Bardov, I.V. Sergeta, N.E. Bacherikov, M.P Vorontsov. and others. 
The basis of this theory is the concept of preclinical diagnosis, which is a system of thought and actions aimed at establishing and correction of borderline states in the context of their occurrence under the influence of environmental and inherited risk factors.

Over the past 20 years, the idea took shape to the theory. It has been confirmed by numerous laboratory and field experiments, which held in KhNMU on the base of interdepartmental integration (Department of Interior and Occupational Diseases, Department of biochemistry, Department of hygiene and ecology No.1 and No.2, Research Institute of Occupational Hygiene and Occupational Diseases, Central Scientific Research Laboratory). Five subjects were carried out in this direction with the priority funding of the Ministry of Health of Ukraine.

Scientific product obtained as a result of all these innovative researches allowed to come to a new understanding of the laws of pathogenesis and to evaluate health and disease as a critical manifestation of the same process, i.e. sanogenesis with a broad area of prenosological states in between.

To determinate the whole complex of diagnostic, preventive and corrective measures aimed to reduce the risk and prevent diseases of different etiology, we have introduced an essentially new term, namely medicine of borderline conditions.

Ensuing in connection with this radical change in our ideas about the formation of health inevitably led to a revision of the existing paradigm of health care. Change of its concept one.

The traditional model of health care based on a clear leaving no alternative understanding of categories "health" and a "disease", which implies an abrupt transition from one state to another: from physiological to pathological state (figure 1). But, as experience shows, this is not so. The development of clinical forms of the disease is preceded by a whole range of functional disorders that occur on the background of quite specific, measurable environmental and organismic complex of risk factors. 
Traditional methodology has led to unnecessary separation of medicine in the preventive and clinical fields. This, in turn formalized and castrated the term "health care facility" (making them actually "clinics"), and led to the reform of sanitaryepidemiological service (through the actual elimination). Ultimately, we traditionally do not treat the patient but the disease. Nosological unit by the stereotyped protocols.

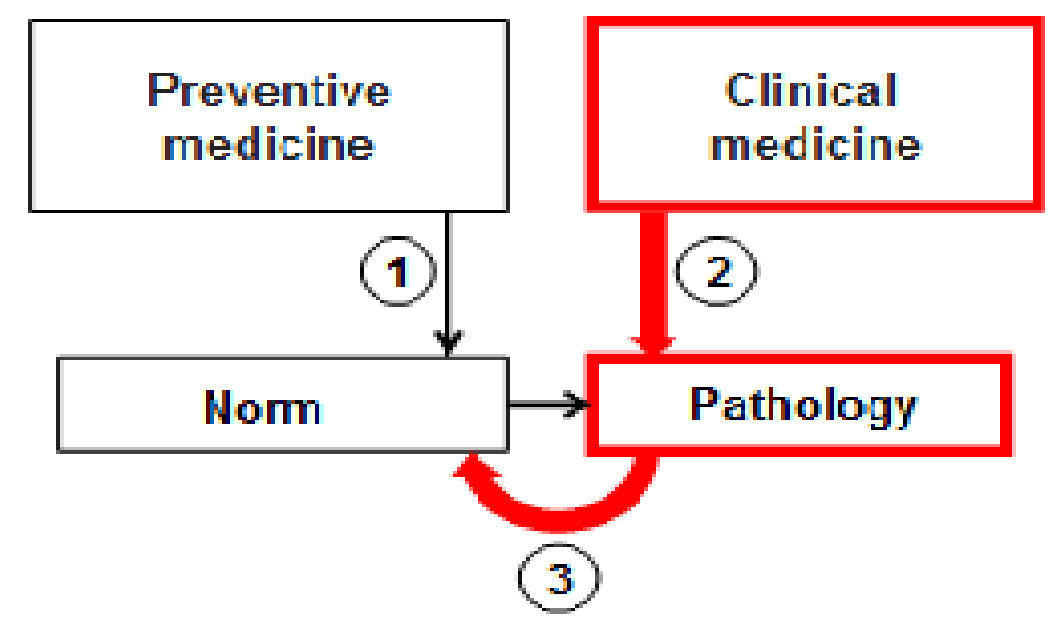

Fig.1 The existing paradigm of health care

(1 - Detection and elimination of risk factors; 2 - Clinical diagnostics, treatment and rehabilitation; 3 - Health restoration)

The new health care paradigm, based on the theory of medicine of borderline states, states that the development of the clinical forms of somatic and mental illnesses is preceded well-defined functional disorders of prenosological character. These deviations can be measured, evaluated and systematized.

This timely detection of borderline conditions, identification and elimination of risk factors for their occurrence and their medical correction contribute to the preservation and strengthening of health. And the cost of prenosological diagnosis is incomparably less than the entire range of therapeutic, diagnostic and rehabilitation measures in the case of illness development. 
This is a new paradigm of medicine that provides a displacement of emphasis from the concepts of "norm" and "pathology" to the concept "prenosology" (figure 2).

The implementation of the proposed concept provides the realization of a number of mandatory common requirements: 1) the mass health examination of the population, 2) monitoring of health at the individual, population and state levels, 3) holding the address (if indicated) preventive measures. All this is in full compliance with European model of health care and with the principles of insurance medicine.

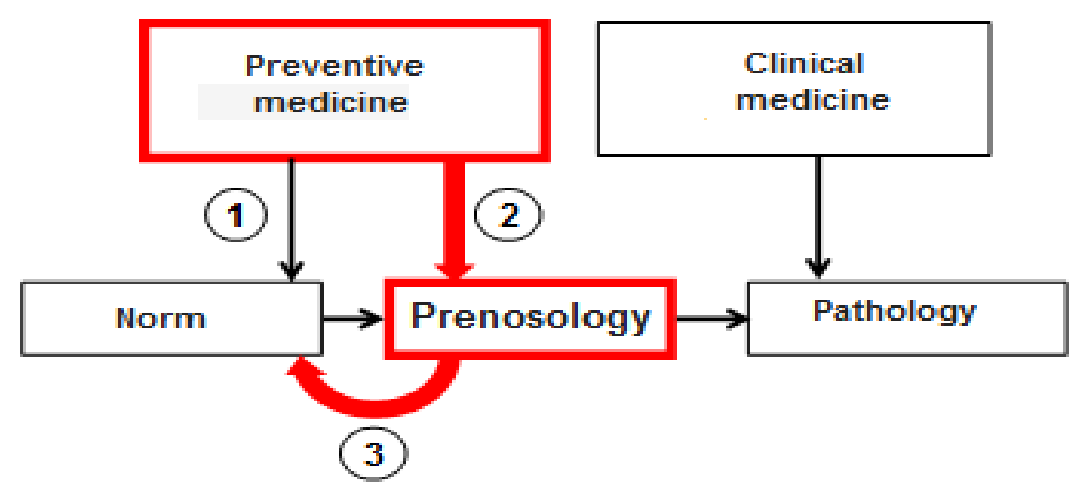

Fig.1 The new paradigm of health care

(1 - Detection and elimination of risk factors; 2 -

Prenosological diagnostics and correction of functional state;

3 - Health restoration)

Most importantly, the proposed new paradigm of health care is focused not on the patient but on a healthy person, and thus fills the real meaning of the term "health care".

Thus, today medicine of borderline conditions is the most progressive and perspective health management model. A fundamentally new concept, i.e. targeted prevention based on diagnostics and correction of prepathological states lies in its basis.

To date, the materials directly related to these issues have been published in more than 200 published works, being included in monographs, textbooks, issued in 
the form of newsletters and methodical guidelines; protected by patents for inventions. Dissertations on this subject are being written or are planned to be written. Our views are shared by our scientific partners, representatives of medical schools from various educational and research institutions of Lugansk, Kiev, Vinnitsa, Lodz, Frankfurt and other cities and countries.

At the same time active promotion of the new ideas in the national and global professional community is the primary method of changing the outdated paradigm of health.

In this connection, we consider it expedient:

1) to propose the Ministry of Health of Ukraine the concept of medicine of borderline conditions developed in KhnNMU as the methodological basis of the reform of the national health care;

2) to develop organizational and methodical mechanisms for the implementation of the principles of medicine of borderline conditions in public health practice (Kharkiv City Department of Health and the Regional State Administration, Department of Social Medicine, Organization and Health Care Economics).

3 ) to continue the elaboration of theoretical foundations of medicine of borderline conditions and practical application of prenosological diagnosis methods in health care practice (theoretical and clinical departments of KhNMU).

Received: 17.12.2015

Accepted: 28.12 .2015 